\title{
The Prolongation Problem for the Heavenly Equation
}

\author{
M. Palese * \\ Department of Mathematics, University of Turin \\ Via C. Alberto 10, 10123 Turin, Italy \\ E-mail: PALESE@DM.UNITO.IT \\ and \\ R.A. Leo, G. Soliani if \\ Department of Physics, University of Lecce \\ Via Arnesano, 73100 Lecce, Italy \\ E-mail: LEORA@LE.INFN.IT, SOLIANI@LE.INFN.IT
}

\begin{abstract}
We provide an exact regular solution of an operator system arising as the prolongation structure associated with the heavenly equation. This solution is expressed in terms of operator Bessel coefficients.
\end{abstract}

1991 MSC: 83C20,35A30,58G35,33C10.

Key words: heavenly equation, prolongation structures, special functions.

\section{Introduction}

It is well known that the so-called heavenly equation

$$
u_{x x}+u_{y y}+\left(e^{u}\right)_{z z}=0,
$$

where $u=u(x, y, z)$ and subscripts mean partial derivatives, occurs in the study of heavenly spaces (Einstein spaces with one rotational Killing vector) [3, 5, 6, 0, 10]

*Speaker at the Conference. Partially supported by GNFM of CNR, MURST, University of Turin.

${ }^{\dagger}$ Partially supported by INFN, MURST, University of Lecce. 
and in the context of extended conformal symmetries [9]. Reduced versions of Eq. (1) have been found in [1] via the symmetry approach, providing instanton and meron-like configurations. Furthermore, an algebra of the Virasoro type without central charge was associated with the heavenly equation by resorting to its invariance under conformal transformations [1]].

To investigate the algebraic aspects of the integrability properties of Eq. (11), we shall apply the prolongation technique, which could provide the relative linear spectral problem [4, 8, 11, 12]. In more than two independent variables, the extension of the prolongation procedure is generally nontrivial and some aspects remain to be explored (see, for example, [8, 11]). However, the main result of this note is represented by the exact solution of the prolongation system associated with Eq. (1). This system consists of three operator equations in the form of commutator relations which can be written as second order (operator) differential equations resembling formally equations of the Bessel type. Our solution is achieved through a series expansion defining operator Bessel coefficients.

\section{The prolongation structure}

Let us introduce on a manifold with local coordinates $(x, y, z, u, p, q, r)$ the closed differential ideal defined by the set of 3-forms:

$$
\begin{aligned}
\theta_{1}= & d u \wedge d x \wedge d y-r d x \wedge d y \wedge d z \\
\theta_{2}= & d u \wedge d y \wedge d z-p d x \wedge d y \wedge d z \\
\theta_{3}= & d u \wedge d x \wedge d z+q d x \wedge d y \wedge d z \\
\theta_{4}= & d p \wedge d y \wedge d z-d q \wedge d x \wedge d z+ \\
& e^{u} d r \wedge d x \wedge d y+e^{u} r^{2} d x \wedge d y \wedge d z
\end{aligned}
$$

where $\wedge$ stands for the exterior product.

It is easy to verify the following

Proposition 2.1 On every integral submanifold defined by $u=u(x, y, z), p=$ $u_{x}, q=u_{y}, r=u_{z}$, with $d x \wedge d y \wedge d z \neq 0$, the ideal (9)-(5) is equivalent to Eq. (1).

Now let us consider the 2-forms:

$$
\begin{aligned}
& \Omega^{k}=H^{k}\left(u, u_{x}, u_{y}, u_{z} ; \xi^{m}\right) d x \wedge d y+F^{k}\left(u, u_{x}, u_{y}, u_{z} ; \xi^{m}\right) d x \wedge d z \\
& +G^{k}\left(u, u_{x}, u_{y}, u_{z} ; \xi^{m}\right) d y \wedge d z+A_{m}^{k} d \xi^{m} \wedge d x+B_{m}^{k} d \xi^{m} \wedge d z+d \xi^{k} \wedge d y
\end{aligned}
$$


where $\xi=\left\{\xi^{m}\right\}, k, m=1,2, \ldots, \mathrm{N}$ (N arbitrary), and $H^{k}, F^{k}$ and $G^{k}$ are, respectively, the pseudopotential and functions to be determined. Furthermore, the quantities $A_{m}^{k}$ and $B_{m}^{k}$ denote the elements of two $N \times N$ constant regular matrices, and the summation convention over repeated indices is understood.

Definition 2.1 The forms $\Omega^{k}$ are called the prolongation forms associated with Eq. (1). Let $\mathcal{I}$ be the prolonged ideal generated by the forms $\theta_{j}$ and $\Omega^{k}$. We say that $\mathcal{I}$ is closed if $d \Omega^{k} \in \mathcal{I}\left(\theta_{j}, \Omega^{k}\right)$.

Lemma 2.1 The closure condition for $\mathcal{I}$ yields

$$
\begin{aligned}
& H^{k}=e^{u} u_{z} L^{k}\left(\xi^{m}\right)+P^{k}\left(u, \xi^{m}\right), \\
& F^{k}=-u_{y} L^{k}\left(\xi^{m}\right)+N^{k}\left(\xi^{m}\right), \\
& G^{k}=u_{x} L^{k}\left(\xi^{m}\right)+M^{k}\left(u, \xi^{m}\right),
\end{aligned}
$$

where $L^{k}, P^{k}, N^{k}, M^{k}$ are functions of integration.

Proof. The closure condition is equivalent to the following constraints:

$$
\begin{gathered}
H_{u_{z}}^{k}-e^{u} G_{u_{x}}^{k}=0, \quad F_{u_{y}}^{k}+G_{u_{x}}^{k}=0, \\
H_{u_{x}}^{k}=H_{u_{y}}^{k}=F_{u_{x}}^{k}=F_{u_{y}}^{k}=G_{u_{y}}^{k}=G_{u_{z}}^{k}=0, \\
u_{z} H_{u}^{k}-u_{y} F_{u}^{k}+u_{x} G_{u}^{k}-e^{u} u_{z}^{2} G_{u_{x}}^{k}+[G, H]^{k}=0, \\
F_{\xi^{l}}^{k}-G_{\xi^{m}}^{k} A_{l}^{m}-H_{\xi^{m}}^{k} B_{l}^{m}=0, \\
F_{\xi^{m}}^{k}\left(B^{-1}\right)_{n}^{m} G^{n}-G_{\xi^{m}}^{k}\left(B^{-1}\right)_{n}^{m} F^{n}=0, \\
{[A, B]=0,}
\end{gathered}
$$

where $[G, H]^{k}=G^{j} H_{\xi^{j}}^{k}-H^{j} G_{\xi^{j}}^{k}$ (Lie bracket), $H_{\xi^{j}}^{k}=\frac{\partial H^{k}}{\partial \xi^{j}}, H_{u}^{k}=\frac{\partial H^{k}}{\partial u}$, and so on. Equations (10)-(11) provide the result.

In the following we shall omit the indices $k, m$ for simplicity.

Proposition 2.2 The following prolongation equations hold

$$
\begin{aligned}
& P_{u}=e^{u}[L, M], \\
& M_{u}=-[L, P], \\
& {[M, P]=0 .}
\end{aligned}
$$


Proof. It is a straightforward consequence of Lemma 2.1 (Eq. (12)). $\quad \square E D$

Hereafter, $L, P, M$ will be regarded as (regular) operators, in the sense that

$$
L \rightarrow L^{j} \frac{\partial}{\partial \xi^{j}}, \quad M \rightarrow M^{j} \frac{\partial}{\partial \xi^{j}}, \quad P \rightarrow P^{j} \frac{\partial}{\partial \xi^{j}},
$$

while the Lie brackets become commutators.

\section{Exact solution of the prolongation equa- tions}

Now, let us look for an exact solution to Eqs. (16)-(18).

Definition 3.1 For any $A=A^{j} \frac{\partial}{\partial \xi^{j}}$, we define $\mathcal{L}[\mathcal{A}]$ by

$$
\mathcal{L}[A]=[L, A] .
$$

Definition 3.2 We define the $n$-th power of the operator $\mathcal{L}$ by setting

$$
\mathcal{L}^{n}[A]=[L,[L, \ldots,[L, A] \ldots]
$$

where $L$ appears $n$-times, and $\mathcal{L}^{0}[A]=A$.

Remark 3.1 The prolongation Eqs. (16) and (17) can be written as the second order operator equations

$$
\begin{aligned}
& P_{t t}-\frac{1}{t} P_{t}+\mathcal{L}^{2}[P]=0, \\
& M_{t t}+\frac{1}{t} M_{t}+\frac{1}{t} \mathcal{L}^{2}[M]=0,
\end{aligned}
$$

where $t=2 e^{\frac{u}{2}}$. 
Remark 3.2 Notice that the above equations resemble formally conventional Bessel equations of the type

$$
\kappa_{t t}-\frac{1}{t} \kappa_{t}+\omega^{2} \kappa=0, \quad \chi_{t t}+\frac{1}{t} \chi_{t}+\frac{1}{t} \omega^{2} \chi=0,
$$

with $\omega$ a constant, whose regular solutions at $t=0$ are given by

$$
\kappa(t)=p_{0} \frac{t}{2} J_{1}(t \omega), \quad \chi(t)=m_{0} J_{0}(t \omega),
$$

with $p_{0}, m_{0}$ constants of integration.

We are interested in solutions of Eqs. (21) and (22) which are regular at $t=0$. We proceed in a heuristic way generalizing the scheme working out for the solution of the operator equations:

$$
\tilde{P}_{t t}+\mathcal{L}^{2}[\tilde{P}]=0, \quad \tilde{M}_{t t}+\mathcal{L}^{2}[\tilde{M}]=0,
$$

whose formal solution is

$$
\begin{aligned}
& \tilde{P}(t, \xi)=e^{i t \mathcal{L}}\left[A_{0}(\xi)\right]+e^{-i t \mathcal{L}}\left[B_{0}(\xi)\right], \\
& \tilde{M}(t, \xi)=e^{i t \mathcal{L}}\left[C_{0}(\xi)\right]+e^{-i t \mathcal{L}}\left[D_{0}(\xi)\right],
\end{aligned}
$$

where $A_{0}, B_{0}, C_{0}, D_{0}$ can be determined from the initial conditions.

Definition 3.3 Let $\mathbf{J}_{\mathbf{0}}(\cdot)$ and $\mathbf{J}_{\mathbf{1}}(\cdot)$ be formally defined by

$$
\begin{aligned}
& \mathbf{J}_{\mathbf{0}}\left(t \mathcal{L}\left[M_{0}\right]\right)=\sum_{m=0}^{\infty} \frac{(-1)^{m}}{(m !)^{2}}\left(\frac{t}{2}\right)^{2 m} \mathcal{L}^{2 m}\left[M_{0}\right] \\
& \mathbf{J}_{\mathbf{1}}\left(t \mathcal{L}\left[P_{0}\right]\right)=\sum_{m=0}^{\infty} \frac{(-1)^{m}}{m !(m+1) !}\left(\frac{t}{2}\right)^{1+2 m} \mathcal{L}^{1+2 m}\left[P_{0}\right] .
\end{aligned}
$$

Remark 3.3 Here $\mathbf{J}_{\mathbf{0}}$ and $\mathbf{J}_{\mathbf{1}}$ are borrowed by the series expansion of the Bessel functions $J_{\nu}$, with $\nu$ an integer:

$$
J_{\nu}(\tau)=\sum_{m=0}^{\infty} \frac{(-1)^{m}}{m !(m+\nu) !}\left(\frac{\tau}{2}\right)^{\nu+2 m} \mathcal{L}^{\nu+2 m}\left[P_{0}\right],
$$

for $\nu=0$ and $\nu=1$, respectively (see [13]). 
The following result holds, whose proof is straightforward.

Proposition 3.1 A solution of equations (21) and (29) regular at $t=0$ is given by

$$
P=\frac{t}{2} \mathbf{J}_{\mathbf{1}}\left(t \mathcal{L}\left[P_{0}\right]\right), \quad M=\mathbf{J}_{\mathbf{0}}\left(t \mathcal{L}\left[M_{0}\right]\right),
$$

where $M_{0} \equiv M_{0}(\xi)=\left.M(t ; \xi)\right|_{t=0}$ and $P_{0} \equiv P_{0}(\xi)$ is such that $\left[L, P_{0}\right]=\left[L, M_{0}\right]$.

Remark 3.4 Inserting the operators (29) into Eqs. (21) and (22) yields the condition $\left[\mathcal{L}\left[M_{0}\right], M_{0}\right]=0$.

Remark 3.5 We point out that the search of solutions to Eqs. (21) and (22), regular at $t=0$, is equivalent to tackle the corresponding Cauchy problem with the initial conditions $P(0 ; \xi)=0,\left.P_{t}(t, \xi)\right|_{t=0}=0, M(0 ; \xi)=M_{0},\left.M_{t}(t, \xi)\right|_{t=0}=0$.

For pratical pourposes, e.g. to determine the spectral problem and Bäcklund transformations associated with Eq. (1), we shall rewrite the solutions (29) in a form which contains the operator $L$ and not the operator $\mathcal{L}$. We shall give a regular solution of Eqs. (21) and (22) following a scheme similar to that working out for the case (23).

By induction it is easy to prove the following Lemma.

Lemma 3.1 For $n>0$, (20) takes the form

$$
\mathcal{L}^{n}[A]=\sum_{k=0}^{n}(-1)^{k}\left(\begin{array}{l}
n \\
k
\end{array}\right) L^{n-k} A L^{k} .
$$

As a straightforward consequence we have

$$
\begin{aligned}
& e^{i t \mathcal{L}}\left[A_{0}\right]=\sum_{n=0}^{\infty} \frac{(i t)^{n}}{n !} \sum_{k=0}^{n}(-1)^{k}\left(\begin{array}{l}
n \\
k
\end{array}\right) L^{n-k}\left[A_{0}\right] L^{k} \\
& =\sum_{j=0}^{\infty} \frac{(i t)^{j}}{j !} L^{j}\left[A_{0}\right]\left(\sum_{K=0}^{\infty} \frac{(i t)^{k}}{k !} L^{k}\right) \equiv e^{i t L}\left[A_{0}\right] e^{-i t L},
\end{aligned}
$$

which is just the Baker-Campbell-Hausdorff expansion (see e.g. [2]).

Thus, we can rewrite the solutions of the operator equations (23) in a more suitable form. 


\subsection{Operator Bessel coefficients}

In order to express the solutions (29) of Eqs. (21) and (22) in terms of the operator $L$, we shall introduce operator Bessel coefficients by means of a formal expansion analogous to that used in the case of Bessel functions.

Definition 3.4 Let $X$ be a regular operator. We define the operator Bessel coefficients $\mathbf{J}_{\mathbf{m}}(t X)$, as the coefficients of the formal expansion:

$$
e^{\frac{t}{2} X(z-1 / z)}=\sum_{m=-\infty}^{\infty} z^{m} \mathbf{J}_{\mathbf{m}}(t X) .
$$

Remark 3.6 We stress that the Laurent series on the right side is uniformly convergent.

First we prove a technical Lemma.

Lemma 3.2 Operator Bessel coefficients satisfy the following recurrence and derivation formulae:

$$
\begin{aligned}
& \mathbf{J}_{-\mathbf{k}}(t L)=(-1)^{k} \mathbf{J}_{\mathbf{k}}(t L), \\
& 2 k \mathbf{J}_{\mathbf{k}}(t L)=t L\left[\mathbf{J}_{\mathbf{k}-\mathbf{1}}(t L)+\mathbf{J}_{\mathbf{k}+\mathbf{1}}(t L)\right], \\
& 2 \frac{d}{d t}\left[\mathbf{J}_{\mathbf{k}}(t L)\right]=L\left[\mathbf{J}_{\mathbf{k}-\mathbf{1}}(t L)-\mathbf{J}_{\mathbf{k}+\mathbf{1}}(t L)\right] . \\
& \frac{d}{d t}\left[t^{k} \mathbf{J}_{\mathbf{k}}(t L)\right]=L t^{k} \mathbf{J}_{\mathbf{k}-\mathbf{1}}(t L), \\
& \frac{d}{d t}\left[t^{-k} \mathbf{J}_{\mathbf{k}}(t L)\right]=-L t^{-k} \mathbf{J}_{\mathbf{k}+\mathbf{1}}(t L),
\end{aligned}
$$

Proof. By differentiating with respect to $z$ the formal expansion (32), with $X=L$, we obtain

$$
\frac{1}{2} t L\left(1+\frac{1}{z^{2}}\right) \sum_{k=-\infty}^{\infty} z^{k} \mathbf{J}_{\mathbf{k}}(t L)=\sum_{k=-\infty}^{\infty} k z^{k-1} \mathbf{J}_{\mathbf{k}}(t L) .
$$

Then, by equating coefficients of $z^{k-1}$ in the above identity we obtain formula (34). Furthermore, if we differentiate the formal expansion with respect to $t$ we have

$$
\frac{1}{2} L(z-1 / z) \sum_{k=-\infty}^{\infty} z^{k} \mathbf{J}_{\mathbf{k}}(t L)=\sum_{k=-\infty}^{\infty} z^{k} \frac{d}{d t} \mathbf{J}_{\mathbf{k}}(t L) .
$$

By equating coefficients of $z^{k}$ on either side of this identity we obtain formula (35). Formulae (36) and (37) can be determined by adding and substracting (34) and (35), while Eq. (33) follows directly from (32) and from the hypothesis that $L$ is a regular operator.

$Q E D$ 


\subsection{A form of the solution of the prolongation equa- tions in terms of $L$}

In the following we shall provide an equivalent solution to Eqs. (21) and (22) in terms of $L$ which is in some sense an analogue of formula (31).

Proposition 3.2 We can rewrite the solution (29) in terms of $L$ as follows:

$$
P=\frac{t}{2} \sum_{k=-\infty}^{\infty} \mathbf{J}_{\mathbf{k}+\mathbf{1}}(t L) P_{0} \mathbf{J}_{\mathbf{k}}(t L), \quad M=\sum_{k=-\infty}^{\infty} \mathbf{J}_{\mathbf{k}}(t L) M_{0} \mathbf{J}_{\mathbf{k}}(t L)
$$

Proof. To verify that the operators (38) obey Eqs. (21) and (22), we refer to Lemma 3.2. In fact, by resorting to (36), (37) and (33) we have

$$
P_{t t}=\frac{1}{2} \sum_{k=-\infty}^{\infty}\left[\mathbf{J}_{\mathbf{k}}(t L) \mathcal{L}\left[P_{0}\right] \mathbf{J}_{\mathbf{k}}(t L)\right]+\frac{t}{2} \sum_{k=-\infty}^{\infty}\left[\mathbf{J}_{\mathbf{k}}(t L) \mathcal{L}^{2}\left[P_{0}\right] \mathbf{J}_{\mathbf{k}+\mathbf{1}}(t L)\right]
$$

On the other hand, since

$$
\begin{aligned}
& \mathcal{L}^{2}[P]=-\frac{t}{2} \sum_{k=-\infty}^{\infty}(-1)^{k}\left[\mathbf{J}_{\mathbf{k}}(t L) L^{2} P_{0} \mathbf{J}_{\mathbf{1}-\mathbf{k}}(t L)-2 \mathbf{J}_{\mathbf{k}}(t L) L P_{0} L \mathbf{J}_{\mathbf{1}-\mathbf{k}}(t L)\right. \\
& \left.+\mathbf{J}_{\mathbf{k}}(t L) P_{0} L^{2} \mathbf{J}_{\mathbf{1}-\mathbf{k}}(t L)\right]=-\frac{t}{2} \sum_{k=-\infty}^{\infty}\left[\mathbf{J}_{\mathbf{k}}(t L) \mathcal{L}^{2}\left[P_{0}\right] \mathbf{J}_{\mathbf{k}+\mathbf{1}}(t L)\right]
\end{aligned}
$$

we obtain the result.

In a similar way, by virtue of (34), we can write

$$
L \mathbf{J}_{\mathbf{k}+\mathbf{2}}(t L)=\frac{2(k+1)}{t} \mathbf{J}_{\mathbf{k}+\mathbf{1}}(t L)-L \mathbf{J}_{\mathbf{k}}(t L) .
$$

This, with the help of Lemma 3.2, gives the following expression for $M_{t t}$ :

$$
\begin{aligned}
& -\sum_{k=-\infty}^{\infty}\left[\mathbf{J}_{\mathbf{k}}(t L) \mathcal{L}^{2}\left[M_{0}\right] \mathbf{J}_{\mathbf{k}}(t L)\right]+\sum_{k=-\infty}^{\infty}(-1)^{k} 2(k-1)\left[\mathbf{J}_{\mathbf{k}-\mathbf{1}}(t L) L M_{0} \mathbf{J}_{-\mathbf{k}}(t L)\right. \\
& \left.-2 \mathbf{J}_{\mathbf{k}-\mathbf{1}}(t L) M_{0} L \mathbf{J}_{-\mathbf{k}}(t L)+\mathbf{J}_{-\mathbf{k}}(t L) M_{0} L \mathbf{J}_{\mathbf{k}-\mathbf{1}}(t L)\right] .
\end{aligned}
$$

Then, using the expression

$$
\mathcal{L}^{2}[M]=\sum_{k=-\infty}^{\infty}\left[\mathbf{J}_{\mathbf{k}}(t L) \mathcal{L}^{2}\left[M_{0}\right] \mathbf{J}_{-\mathbf{k}}(t L)\right]
$$


and taking into account (33) and (34), we have

$$
\begin{aligned}
& t M_{t t}+M_{t}+\mathcal{L}^{2}[M]=\sum_{k=-\infty}^{\infty}\left[2 k \mathbf{J}_{\mathbf{k}-\mathbf{1}}(t L) L M_{0} \mathbf{J}_{\mathbf{k}}(t L)+\right. \\
& \left.-2(k-1) \mathbf{J}_{\mathbf{k}-\mathbf{1}}(t L) M_{0} L \mathbf{J}_{\mathbf{k}}(t L)\right]=0 .
\end{aligned}
$$

Hence Eq. (22) is satisfied. This completes the proof.

\section{Conclusions}

We have solved the prolongation problem for Eq. (1) in terms of a series expansion of operators which can be interpreted as generalized Bessel coefficients. The operators (38) have been derived under the hypothesis that $P$ and $M$ are regular at $t=0$, i.e. at $u \rightarrow-\infty$. The "key" for our result is based on Eqs. (21) and (22), which constitute an extended form (of operator Bessel type) of the operator equations (23). We point out that the introduction of the operator (30) has strongly facilited our task. To this regard, a useful step is represented by Eq. (31), where a correspondence is established between the operators $\mathcal{L}$ and $L$ via the Baker-Campbell-Hausdorff formula.

In theory, the knowledge of $H, F$ and $G$ (see (7)-(9) ) may be exploited to find the spectral problem related to Eq. (1). However, in opposition to what happens in other cases, the determination of the spectral problem of Eq. (11) within the prolongation scheme offers notable difficulties, mainly owing to the fact that we have been able to get only a solution of the prolongation equations (16)-(18) which is regular at $t=0$. At present the construction of a prolongation algebra whose elements depend uniquely on the pseudopotential variables remain an open problem.

\subsection{Acknowledgments}

One of us (M. P.) would thank M. Ferraris and M. Francaviglia for useful discussions.

\section{References}

[1] Alfinito, E., Soliani, G., and Solombrino, L.: The symmetry structure of the heavenly equation Lett. Math. Phys. 41, 379-389 (1997). 
[2] Baker, H.F.: Alternant and continuous group, Proc. London Math. Soc. (2) 3, 24-47 (1904); Campbell, J.E.: On a law of combination of operators, Proc. London Math. Soc. 29, 14-32 (1898); Hausdorff, F.: The symbolic exponential formula in group theory, Ber. Verh.Sächs. Gess. Wiss. Leipzig. Math.-Phys. Kl. 58, 19-48 (1906).

[3] Boyer, C. and Finley, J.D.: Killing vectors in self-dual, Euclidean Einstein spaces, J. Math. Phys. 23, 1126-1128 (1982).

[4] Estabrook, F.B. and Wahlquist, H.D.: Prolongation structures of nonlinear evolution equations. II, J. Math. Phys. 17, 1293-1297 (1976).

[5] Gegenberg, J.D. and Das, A.: Stationary Riemannian space-times with self-dual curvature, Gen. Relativity and Gravitation 16, 817-829 (1984).

[6] Gibbons, G.W. and Hawking, S.W.: Gravitational Multi-Instantons, Phys. Lett.B78, 430-432 (1978).

[7] Lebrun, C.: Explicit self-dual metrics on $C P_{2} \# \ldots \# C P_{2}$, J. Diff. Geom. 34, 223 (1991).

[8] Morris, H.C.: Prolongation structures and nonlinear evolution equations in two spatial dimensions, J. Math. Phys. 17, 1870-1872 (1976).

[9] Park, Q-Han: Extended conformal symmetries in real heavens, Phys. Lett. 236B, 429-432 (1990).

[10] Plebanski, J.F.: Some solutions of complex Einstein equations J. Math. Phys. 16, 2395-2402 (1975).

[11] Tondo, G.S.: The eigenvalue problem for the Three-Wave Resonant Interaction in $(2+1)$ dimensions via the prolongation structure, Lett. Nuovo Cimento 44, 297-302 (1985).

[12] Whalquist, H.D. and Estabrook, F.B.: Prolongation structures of nonlinear evolution equations, J. Math. Phys. 16, 1-7 (1975).

[13] Watson, G.N.: A Treatise on the Theory of Bessel Functions, Cambridge University Press, Cambridge, 1952. 